strains ${ }^{10,11}$. These findings make it unlikely that mutations or structural alterations of haem polymerase can account for chloroquine resistance. A mechanism analogous to the multiple drug resistance $(m d r)$ mechanism in mammalian tumour cells has been proposed to account for the rapid-efflux resistant phenotype ${ }^{11}$ Two $P$. falciparum genes with homologies to mammalian $m d r$ genes were not, however, linked to chloroquine resistance in a genetic cross, and exceptions to an association of resistance with point mutations in an $m d r$-like gene were also apparent in a population survey ${ }^{12,13}$. Linkage studies instead place the determinant of the rapid-efflux, chloroquineresistance mechanism within a segment of $P$. falciparum chromosome 7 (ref. 14).

The loss of chloroquine as a safe, cheap and effective remedy for malaria already threatens to overwhelm the beleagured medical services of many countries. Understanding the molecular foundations of chloroquine action and chloroquine resistance - processes that seem to have distinct mechanisms may point to ways to modify existing quinoline-ring structures, develop therapeutically useful reversal agents or design new classes of haem polymerase inhibitors. In haem polymerization, the parasite is showing us a vulnerable target. The challenge is to find ways to hit it again.

Thomas E. Wellems is in the Laboratory of Parasitic Diseases, National Institute of Allergy and Infectious Diseases, Bethesda, Maryland 20892, USA.

\title{
Who needs peptide transporters?
}

\section{Bernhard Dobberstein}

THE discovery of genes in the major histocompatibility complex (MHC) that code for subunits of proteasomes and putative ATP-driven transmembrane transporters has led to a great deal of speculation about their involvement in antigen processing and peptide binding to MHC class I molecules (the developments were discussed at the time in News and Views ${ }^{1,2}$ ). The most favoured hypothesis is that proteasomes digest cytosolic proteins into fragments which are then, in an ATP-dependent step, translocated across the membrane of the endoplasmic reticulum (ER) by the transporters. Writing in Cell, Lévy et al. ${ }^{3}$ challenge this view. They report that ATP is required for the binding of peptide to MHC class I molecules rather than for peptide transport into the lumen of the ER.

MHC class I molecules are composed of two subunits, a membrane-spanning heavy chain and $\beta_{2}$-microglobulin, a small exoplasmic protein. Heavy chains are polymorphic and encoded by genes in the MHC. Shortly after their synthesis, heavy chains assemble noncovalently with $\beta_{2}$-microglobulin in the ER. Binding of peptides, nine or ten amino-acid residues in length, stabilizes the association between heavy chain and $\beta_{2}$-microglobulin and makes the complex competent for transport to the cell surface, where MHC class I molecules activate cytotoxic $\mathrm{T}$-cells by presenting peptides to them ${ }^{4}$.

Several mutant cell lines have been characterized in which MHC class I molecules are synthesized but not expressed on the cell surface. Only after the addition of peptides are these molecules found on the cell surface ${ }^{4,5}$. In one cell line the defect could be repaired by transfection with a gene encoding a member of the superfamily of ATPdriven transporter proteins ${ }^{6}$. It was therefore proposed that peptide transport from the cytosol into the lumen of the ER is an active, ATP-dependent process. The experiments now described by Lévy et al. ${ }^{3}$ aim at a direct demonstration of the participation of ATPdriven transporters in supplying class I molecules with peptide.

An in vitro system was used in which class I heavy chains were synthesized and cotranslationally inserted into membranes derived from the rough ER of lymphoblastoid cells. The membranes, also called rough microsomes, form spherical vesicles that are impermeable to proteases added from the outside. Microsomes have been widely used to study the translocation of nascent secretory proteins. This process requires the presence of an ER signal sequence on the nascent polypeptide, GTP, possibly also ATP, and several cytosolic and membrane factors ${ }^{7}$. Most secretory proteins can be translocated only cotranslationally, but some small ones with relative molecular masses of about $6,000-8,000\left(M_{\mathrm{r}} 6-8 \mathrm{~K}\right)$ are also translocated post-translationally ${ }^{8}$. Microsomal membranes were generally found to be impermeable to proteins lacking a signal sequence, but small peptides, such as those that are substrates for an ER oligosaccharyl transferase ${ }^{9}$, can pass through the membrane. When added to cells or microsomes, peptides with 3-5 amino-acid residues are found inside the lumen of the ER or of microsomes, respectively, and become glycosylated. The efficiency of membrane permeation strongly depends on the size and physical properties of the peptides. How these peptides cross the ER membrane is unclear.

To test whether ATP-binding proteins are involved in peptide transport across the ER membrane, Lévy et al. ${ }^{3}$ translated messenger RNA coding for class I heavy chains in a reticulocyte lysate supplemented with rough microsomes from the lymphoblastoma cell line Raji. The microsomes contain endogenous $\beta_{2}$-microglobulin. After translation, ATP was depleted and a peptide from the nucleoprotein influenza A (NP 384-394) in its biotinylated form was added ${ }^{10}$. In the presence of ATP, the peptide stabilized the oligomeric assembly of heavy chains with $\beta_{2}$-microglobulin and bound to this complex. Depletion of ATP prevented the assembly of heavy chains with $\beta_{2}$-microglobulin and prevented the binding of peptide. In the absence of ATP, peptide was bound by a chaperone (BiP, the human immunoglobulinbinding protein) and released from it in the presence of ATP; BiP is a soluble heat-shock protein analogue of the ER lumen $^{11}$, and has been shown to interact with partially folded and misfolded proteins in an ATP-sensitive fashion. The

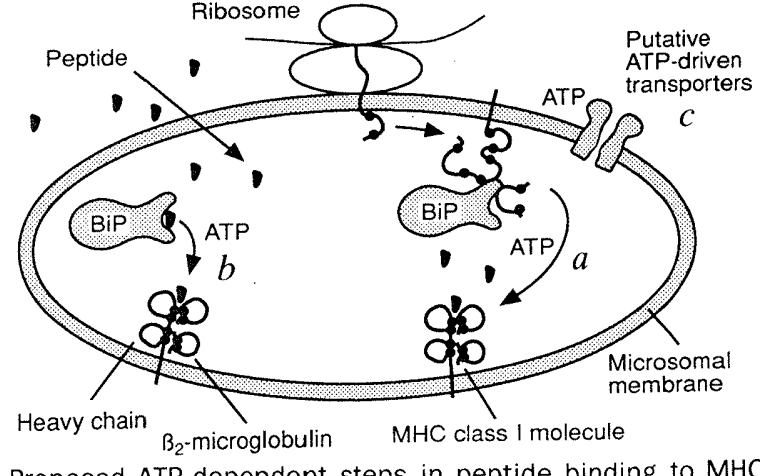

Proposed ATP-dependent steps in peptide binding to MHC class I molecules. The most favoured view is (a) that ATP is chains and $\beta_{2}$-microglobulin so that peptide can bind. The chaperone $\mathrm{BiP}$ is thought to catalyse this process. The alternative view ${ }^{3}$ is $(b)$ that peptide is concentrated by the binding to BiP and that the ATP is required for the release of the peptide. Whether ATP is also required for membrane translocation $(c)$ of peptides derived from degradation of cytosolic proteins remains an open issue.

two experiments convincingly demonstrate that binding of peptide to $\mathrm{MHC}$ class I molecules occurs only in the presence of ATP, and that at least some peptides can enter the lumen of microsomal vesicles in the absence of ATP.

To see whether cytoplasmic domains of transmembrane proteins are involved in peptide transport, Lévy et al. incu- 


\section{RESUME}

\section{Cost of the charge}

SEQuestering a charged side-chain in the low-dielectric-constant heart of a globular protein has always been surmised to exact a ruinous

thermodynamic cost. Sometimes pared charges have evolved to reduce this penalty. Now S. Dao-pin et al.

(Biochemistry 30,11521 11529; 1991) have made two mutants of T4 phage lysozyme, one with lysine for methionine on the inward facing surface of an $\alpha$ helix, the other with aspartate for a leucine. The conformations are less stable, but both chains fold, giving products with 35 per cent and 4 per cent activity. The first mutant has been crystallized and the violated helix is appreciably wobbly. The pk of the intruding lysine is 6.5 and the structure survives down to $\mathrm{pH} 3$. Such mutations, then, are evidently not as calamitous as has been supposed.

\section{Old story}

CONVENTIONAL thinking has it that clonally reproducing organisms don't last for long in evolutionary terms, at least compared with the sexually reproducing stock from which they stem. Ain't necessarily so, find J. M. Quattro and colleagues in their estimation of the time over which a unisexual, all-female lineage of fish has persisted (Proceedings of the National Academy of Sciences 89, 348-352; 1992). Quattro et al. looked at 'post-formational' (that is, post-hybridization) mutations in the mitochondrial DNA and allozymes of the unisexual, live-bearing fish Poeciliopsis monacha-occidentalis, which occurs in arroyos (streams) in Mexico where the distributions of its two sexual progenitors overlap. As evidenced by mitochondrial DNA restriction-site data, the lineage manifests a high level of genetic diversity and is, the authors estimate, at least 100,000 generations old.

\section{Steps in time}

L. R. BRAND, together with Thu Tang, has carried out a further appraisal of how the fossil tetrapod trackways in the Coconino sandstone of northern Arizona came to be formed (Geology 19, 1201$1204 ; 1991)$. At issue is whether the sandstone, which is of Permian age, was deposited subaerially, as sand dunes, or under water. Brand and Tang produced diagrams of the orientation of the limb prints and direction of the fossil tracks, and compared them with those made by newts on sand in a tank of flowing water $4 \mathrm{~cm}$ deep. In many of the fossil tracks. limb impression and track direction are at different angles, a feature also evident in the impressions left by the living newts as they were drifted by the current. That, say the authors, points towards an underwater origin for at least some of the sandstone. bated microsomes wh protense. This treatment, the authors argue, should have cleaved the cytoplasmically exposed ATP-binding domain of the postulated transporters. Because no effect on peptide binding to $\mathrm{MHC}$ class I molecules was evident, they concluded that transporters are not involved in this process. But because the protease sensitivity of the putative transporters is not known, the results are suggestive rather than conclusive.

The idea that ATP-driven transporters are involved in the transport of peptides across the ER membrane arose from analysis of mutant cells defective in surface expression of class I molecules. Mutant $T 2$ cells derived from the $B$ lymphoblastoid cell line $\mathrm{T} I$ have a deletion in the MHC, and they express class I molecules intracellularly but not on the cell surface. Cerundolo et al. ${ }^{5}$ found that these cells had lost the ability to present intracellular antigen efficiently, whereas they could present extracellular antigen of eight and twelve amino-acid residues efficiently. One possible explanation for the defect in these cells is that peptides derived from cytosolic proteins do not reach the lumen of the $E R$. To test this possibility, Levy et al. investigated the ability of microsomes from $T 1$ and $T 2$ cells to take up peptides and assemble them with class I molecules. They found that peptides were taken up into microsomes from $\mathrm{T} 1$ and $\mathrm{T} 2$ cells with similar efficiencies. But peptide-stimulated assembly of class I molecules was only seen with microsomes of $\mathrm{T} 1$ cells, not with those of mutant $\mathrm{T} 2$ cells. Levy et al. propose that the defect is in luminal factors needed for peptide binding to the class I molecules.

The authors consider several explanations for the defect in the lumen of microsomes from T2 cells and the ATP requirement for peptide binding to class I molecules. Peptides may become concentrated by binding to BiP and the ATP is needed to release them (see figure). I consider this to be unlikely, because peptide would re-enter the free-peptide pool after release from BiP. Peptides may require trimming in the lumen of the ER before binding to class I molecules. This possibility remains open, however, and needs further experiments with peptides of different size.

Another possibility is that the oligomeric assembly of $\mathrm{MHC}$ class I molecules needs a catalyst for proper folding. This is the most likely explanation for an ATP-dependent step, as experimental evidence for a role of ATP for folding and disulphide-bond formation in proteins in the ER will shortly be described by Braakman and colleagues ${ }^{12}$. These authors demonstrate that folding of a viral glycoprotein in the ER is an energy-dependent process. In the absence of ATP prowem mistolu into drulphide cross-linked aggregates but they can be rescued and fold comecty when cells are allowed to regenerate ATP again. Class 1 heavy chains and $\beta_{2}$-microglobulin may also require ATP for their folding and oligomerization, and may aggregate in the absence of ATP. Under such conditions peptide binding would not occur.

Is there really no requirement for peptide transporters? Indeed for peptides up to about ten amino acids in length there may be no requirement for active transport across microsomal membranes. But a difference in the permeability of small peptides across membranes of isolated microsomal vesicles from that of the ER membrane in intact cells cannot be ruled out. It will be interesting to see whether peptides applied to the cytosol of cells by either micro-injection or after permeabilization of the plasma membrane can cross the ER membrane in an ATP-independent manner.

The results of Lévy et al. raise another important question. What size are the peptides present in the cytosol? In particular, are they the right size to interact with MHC class I molecules? Little is known about the peptide intermediates in the degradation of cytosolic proteins. It is conceivable that proteasomes proteolytically process cytosolic proteins to fragments which can be actively transported across the ER membrane. To draw a more definite conclusion about the requirement for peptide transporters, intact protein substrates, not just trimmed peptides, should be investigated for their requirements for proteolytic processing and membrane translocation. With the in vitro system used by Lévy et al., it should be possible to analyse the entire pathway of proteolytic processing of cytosolic proteins, transport of peptides across the ER membrane and binding to the MHC class I molecules. It is likely that several energy-requiring steps and factors mediating charging of $\mathrm{MHC}$ class I molecules with peptides still await discovery.

Bernhard Dobberstein is in the European Molecular Biology Laboratory, Meyerhofstrasse 1, 6900 Heidelberg, Germany.

1. Robertson, M. Nature 353, 300-301 (1991)

2. Parham, P. Nature 348, 674-675 (1990)

3. Lévy, F., Gabathuler, R., Larsson, R. \& Kvist, S. Cell 67, 265-274 (1991).

4. Neefjes, J. J.. Schumacher, N. M. \& Ploegh, H. L. Current Biol. 3, 601-609 (1991).

5. Cerundolo, V. et al. Nature 345, 449452 (1990).

6. Spies, T. \& DeMars, R. Nature 351, 323-324 (1991)

7. Gilmore, R. Current Biol. 3, 580-584 (1991).

8. Wiech, H., Stuart, R. \& Zimmermann, R. Seminars Cell Biol. 1, 55-63 (1990).

9. Lau, J. T. Y. et al. J. biol. chem. 258, 15255-15260 (1983).

10. Lévy, F., Larsson, R. \& Kvist, S. J. Cell Biol. 115, 959-970 (1991).

11. Pelham, R. B. A. Rev. Cell Biol. 5, 1-23 (1989).

12. Braakman, I., Helenius, J. \& Helenius, A. Nature (in the press).
praakman,

NATURE - VOL 355 -9 JANUARY 1992 